\title{
The relevance of imaging and analytical electron microscopy in the understanding of heterogeneous selective oxidation catalysis
}

\author{
Robert Schlögl
}

Fritz-Haber-Institut der MPG, Faradayweg 4-6, 14195 Berlin

Catalysis is the science and technology of modifying the velocity of chemical transformations. Spatio-temporal decoupling of elementary step reactions that are necesary to transform the starting molecules into products enables this. Selective chemisorptions and diffusion processes are required to perform the individual steps. Thus, catalysis is a surface phenomenon and the bulk of the catalyst material has at first sight little to do with its desired function. It may be asked how in such a situation electron microscopy which is by enlarge a bulk technique can be useful.

The obvious answer is that many catalysts are nanostructured as deposited cluster systems or as microstructured - mesostructured solids with high "internal" surface and that morphological inspection, local crystallography and analytical-spectroscopic information from individual objects of a catalyst material are of great value in understanding and optimising the functional properties of the material.

Selective oxidation catalysts are used to transform small organic molecules of low chemical functionality into derivatives with higher functionality either by abstracting protons and/or by adding oxygen atoms in various geometries to the carbon skeleton. These reactions form the backbone of all polymer industry as they provide the reactive monomer blocks. In smaller volumes but equal importance these reactions form about half of all industrial molecular transformations. Despite of their large relevance, the overall function of the catalyst is far from satisfactory with respect to selective performance and general applicability.

In understanding the application problems it occurs that the role of the bulk structure for the surface process is rather unclear in a class of materials operating as massive (oxides) materials with little geometric surface and with no apparent nanostructuring. The diffusion of lattice oxygen as active component in the catalytic process plays a central role in the classical model ideas. Hence, defects, shear structures and other aspects of microstructure are of relevance. As all these reactions require also the exchange of electrons between the reactants the chemical valence and the local electron density are additional factors controlling the function.

Electron microscopy an in particular analytical electron microscopy as ELNES and X-ray emission spectrometry are very relevant in elucidating the electronic structure and chemical composition of the usually complex multi-phase-multi-element catalytic materials. Often on-top and side-on views onto the surfaces of catalysts are possible revealing directly the interfacial properties with the gas phase and between the grains of the material.

The dynamical nature of both oxygen content and electronic structure of active oxidation catalysts bears the massive problem of strong effects of the electron beam-matter interaction. Imaging artefacts both in structure and morphology are frequent and require very special attention. Modern low-dose 
imaging techniques and calibrated instruments allow to systematically evaluate beam artefacts at least for chemically simple model systems such as binary transition metal oxides.

The dynamical character of the structure of oxidation catalysts calls further for in-situ observations. In these studies elevated ranges of temperature and gas pressure are used to bring the catalyst in an operative state and to determine then its structure. Besides the apparent fundamental problems of high pressure electron microiscopy a high risk of extreme beam damage effects due to the additional presence of low energy secondary charged species and due to the combined chemical-beam interaction on the catalyst require extreme care in assigning general relevance to such observations.

ELNES is a most valuable tool in determining locally the electronic structure of a catalyst if its spectral signatures can be analysed in terms of chemical valence and co-ordination. To this end highresolution data and a sound theoretical understanding are equally essential. Both ingredients allow today in selected cases a much more detailed analysis than fingerprinting reference structures that is, however, a most versatile and quantitatively useful application. High resolution ELNES data may be directly correlated with the corresponding low energy NEXAFS data that can be detected at synchrotron radiation sources under in-situ conditions with surface-sensitive secondary electron detection schemes. In this way highly local bulk information can be matched to extremely surface sensitive detection of the same physical property namely the unoccupied density of states in the valence region. In this way the bulk method ELNES and the surface method NEXAFS can be brought to co-incidence.

The contribution illustrates the points made with a single example of vanadium oxides used selective oxidation of n-butane with air. Reference oxides as clusters and bulk samples, their dynamics in redox chemistry and their beam-damage behaviour will be illustrated. Ternary V-O-P systems will be studied in search of microstructural relations to their catalytic function. The dominating role of beam artefacts in discussing the structure of active samples will be stressed. The combination of electron microscopy and of ELNES-NEXAFS will be used to derive a novel functional model of the system indicating that the bulk oxygen dynamics is only relevant for creating the active phase that can perfectly exert its catalytic function with gas phase ingredients only. This will be contrasted to the function of the same catalyst under transient conditions in which reaction of the organic molecule and of oxygen with the catalyst are separated in time and space.

\section{Reference}

For published work and further details please contact the following url: htttp://w3.rz-berlin.mpg.de/ac/ac.htmil 